\title{
Galois symbol maps for abelian varieties over a $p$-adic field
}

\author{
Toshiro Hiranouchi
}

November 26, 2019

\begin{abstract}
We study the Galois symbol map associated to the multiplicative group and an abelian variety which has good ordinary reduction over a $p$-adic field. As a byproduct, one can calculate the "class group" in the view of the class field theory for curves over a $p$-adic field.
\end{abstract}

\section{Introduction}

Let $k$ be a $p$-adic field, that is, a finite extension of $\mathbb{Q}_{p}$ and its residue field is denoted by $\mathbb{F}$. An objective of the class field theory of a (projective smooth and geometrically connected) curve $X$ over $k$ with function field $k(X)\left([1]\right.$, [15]) is to describe the abelian fundamental group $\pi_{1}^{\mathrm{ab}}(X)$ by an abelian group

$$
S K_{1}(X)=\operatorname{Coker}\left(K_{2}(k(X)) \rightarrow \bigoplus_{x \in X_{0}} k(x)^{\times}\right)
$$

(for the precise definition of $S K_{1}(X)$, see (7)) through the reciprocity map

$$
\rho: S K_{1}(X) \rightarrow \pi_{1}^{\mathrm{ab}}(X)
$$

(cf. Thm.2.4). The "geometric part" $\pi_{1}^{\mathrm{ab}}(X)^{\mathrm{geo}}:=\operatorname{Ker}\left(\pi_{1}^{\mathrm{ab}}(X) \rightarrow \pi_{1}^{\mathrm{ab}}(\operatorname{Spec}(k))\right)$ is approximated by

$$
V(X)=\operatorname{Ker}\left(\partial: S K_{1}(X) \rightarrow k^{\times}\right),
$$

where $\partial$ is defined by the tame symbols. It is known that the induced map $\tau: V(X) \rightarrow \pi_{1}^{\mathrm{ab}}(X)^{\mathrm{geo}}$ from $\rho$ has the finite image and the kernel is the maximal divisible subgroup $V(X)_{\text {div }}$ of $V(X)$. In particular, we have a decomposition

$$
V(X)=V(X)_{\mathrm{div}} \oplus V(X)_{\mathrm{fin}},
$$

for some finite group $V(X)_{\text {fin. }}$. Now we further assume that $X$ has good reduction and also $X(k) \neq \emptyset$. Recall that $X$ said to have good reduction if the special fiber $\overline{\mathscr{X}}:=\mathscr{X} \otimes_{O_{k}} \mathbb{F}$ of the regular model $\mathscr{X}$ over $O_{k}$ with $\mathscr{X} \otimes_{O_{k}} k \simeq X$ is also a smooth curve over $\mathbb{F}$. The Jacobian variety 
$\operatorname{Jac}(\mathscr{X})$ associated to $\mathscr{X}$ has the generic fiber $J=\operatorname{Jac}(X)$ and the special fiber $\bar{J}=\operatorname{Jac}(\overline{\mathscr{X}})$. In particular, $J$ has good reduction. This group $V(X)_{\text {fin }}$ is related to the $G_{k}$-coinvariant part of the Tate module $T(J)=\lim _{m} J[m]$ as

$$
V(X)_{\text {fin }} \stackrel{\simeq}{\rightarrow} T(J)_{G_{k}} .
$$

In this setting, the prime to $p$-part of $V(X)_{\text {fin }}$ is known well as $V(X) / m \simeq \bar{J}(\mathbb{F}) / m$ for any $m$ prime to $p$ (by S. Bloch, see Prop. 2.5). For the $p$-part, only the finiteness is proved ([1], Prop. 2.4). The aim of this note is to study more explicitly on this $V(X)_{\text {fin }}$ by determining the group structure under the following conditions:

(Rat) $J[p] \subset J(k)$,

(Ord) $\bar{J}$ has ordinary reduction, and

(Ram) $k\left(\mu_{p^{N+1}}\right) / k$ is a non-trivial totally ramified extension, where $\mu_{p^{N+1}}$ is the group of $p^{N+1}$-th roots of unity, and $N=\max \left\{n \mid J\left[p^{n}\right] \subset J(k)\right\}$.

The main result of this note is the following:

Theorem 1.1 (Cor. 4.9). Under the conditions (Rat), (Ord), and (Ram) for $J=\operatorname{Jac}(X)$ as above, we have

$$
V(X)_{\mathrm{fin}} \simeq\left(\mathbb{Z} / p^{N}\right)^{\oplus g} \oplus \bar{J}(\mathbb{F})
$$

where $g=\operatorname{dim} J$.

Since the divisible part $V(X)_{\text {div }}$ is the kernel of the reciprocity map $\tau: V(X) \rightarrow \pi_{1}^{\mathrm{ab}}(X)^{\mathrm{geo}}$ and $\tau$ is surjective in the case where $X$ has good reduction, we obtain the structure of the geometric part of the fundamental group as

$$
\pi_{1}^{\mathrm{ab}}(X)^{\mathrm{geo}} \simeq\left(\mathbb{Z} / p^{N}\right)^{\oplus g} \oplus \bar{J}(\mathbb{F}) .
$$

The key tool to compute the group $V(X)$ is the so called Galois symbol map of the following form

$$
s_{m}: K\left(k ; \mathbb{G}_{m}, J\right) / m \rightarrow H^{2}\left(k, \mu_{m} \otimes J[m]\right),
$$

where $K\left(k ; \mathbb{G}_{m}, J\right)$ is the Somekawa $K$-group which is isomorphic to $V(X)$ and the map $s_{m}$ is constructed by a similar way as the Galois symbol map on the Milnor $K$-group $K_{2}(k) / m \rightarrow$ $H^{2}\left(k, \mu_{m}^{\otimes 2}\right)$ (for the precise construction of the map $s_{m}$, see Definition 2.3).

Finally, we give some remarks on the conditions above. The condition (Ram) is technical. For example, let us consider an elliptic curve $X=E$ over $k=\mathbb{Q}_{p}\left(\mu_{p^{M}}\right)$ for some $M \geq 1$ assuming that $E$ has good ordinary reduction as in $($ Ord). Note that we have $\operatorname{Jac}(E)=E$. From the Weil pairing, we have an inequality $N \leq M$ in general (cf. [18], Chap. III, Cor. 8.1.1), where $N=\max \left\{n \mid E\left[p^{n}\right] \subset E(k)\right\}$. Thus, if $E\left[p^{M}\right] \subset E(k)$ then $N=M$. Consequently, the condition (Ram) above automatically holds. For the case $M=1$ (from the above argument, (Rat) implies (Ram)) and $p=3$, by computing SAGE [2], there are 683 elliptic curve $E_{0}$ over $\mathbb{Q}$ with good ordinary reduction at $p=3, \bar{E}_{0}\left(\mathbb{F}_{3}\right)[3] \neq 0$ and the conductor $<1000$. Among them, 269 curves satisfy $E[3] \subset E(k)$ and hence the condition $(\mathbf{R a m})$, where $E=E_{0} \otimes_{\mathbb{Q}} k$ is the base change of $E$ to $k=\mathbb{Q}_{3}\left(\mu_{3}\right)$. 


\section{Contents}

The contents of this note is the following:

- Section 2; After recalling the definition of the Mackey functor and that of the product for two Mackey functors, we review the class field theory for curves over $p$-adic fields following Bloch [1] and Saito [15].

- Section 3, We determine the image of the Kummer map $A(k) / p^{n} \rightarrow H^{1}\left(k, A\left[p^{n}\right]\right)$ (cf. (4) $)$ associated to an abelian variety $A$ with good ordinary reduction over $k$ (Prop. 3.1). This extends the main theorem in [21] for an elliptic curve. The proof is essentially same as in op. cit.

- Section 4. We show that the Galois symbol map (defined in Def. 2.3) associated to the multiplicative group $\mathbb{G}_{m}$ and an abelian variety $A$ over a $p$-adic field is bijective (Thm.4.2), and determine the group structure of the Somekawa $K$-group $K\left(k ; \mathbb{G}_{m}, A\right)$ (Thm. 4.8). Using the isomorphism $K\left(k ; \mathbb{G}_{m}, J\right) \simeq V(X)(c f .(9))$ for a curve $X$ as in Theorem 1.1 and $J=\operatorname{Jac}(X)$, we obtain the structure of $V(X)_{\text {fin }}$.

\section{Notation}

Throughout this note, we use the following notation

- $k$ : a finite extension of $\mathbb{Q}_{p}$,

- $\mathbb{F}$ : the residue field of $k$, and

- $G_{k}=\operatorname{Gal}(\bar{k} / k)$ : the absolute Galois group of $k$.

For a finite extension $K / k$, we define

- $O_{K}$ : the valuation ring of $K$,

- $\mathbb{F}_{K}$ : the residue field of $K$,

- $U_{K}=O_{K}^{\times}$: the unit group, and

- $U_{K}^{i}=1+m_{K}^{i}$ : the higher unit group.

For an abelian variety over $k$, we define

- $\pi: A \rightarrow \bar{A}$ : the reduction map,

- $\widehat{A}$ : the formal group law of $A$ (cf. [6], Sect. C.2), and

- $A_{1}:=\operatorname{Ker}(\pi: A \rightarrow \bar{A})$ : the kernel of the reduction map $\pi$.

For an abelian group $G$ and $m \in \mathbb{Z}_{\geq 1}$, we write $G[m]$ and $G / m$ for the kernel and cokernel of the multiplication by $m$ on $G$ respectively.

\section{Acknowledgments}

I would like to thank Professor Evangelia Gazaki and Dr. Isable Leal for their valuable comments and encouragement. This work was supported by KAKENHI 25800019. 


\section{Class field theory}

\section{Mackey functors}

Definition 2.1 ( $c f$. [14], Sect. 3). A Mackey functor $\mathscr{M}$ (over $k$ ) (or a $G_{k}$-modulation in the sense of [13], Def. 1.5.10) is a contravariant functor from the category of étale schemes over $k$ to the category of abelian groups equipped with a covariant structure for finite morphisms such that $\mathscr{M}\left(X_{1} \sqcup X_{2}\right)=\mathscr{M}\left(X_{1}\right) \oplus \mathscr{M}\left(X_{2}\right)$ and if

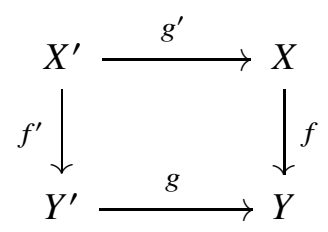

is a Cartesian diagram, then the induced diagram

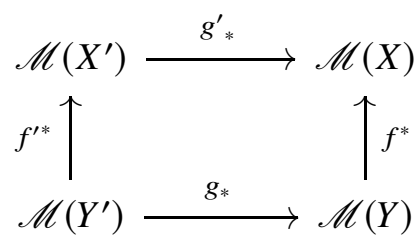

commutes.

For a Mackey functor $\mathscr{M}$, we denote by $\mathscr{M}(K)$ its value $\mathscr{M}(\operatorname{Spec}(K))$ for a field extension $K$ of $k$. For any finite extensions $k \subset K \subset L$, the induced homomorphisms from the canonical map $j: \operatorname{Spec}(L) \rightarrow \operatorname{Spec}(K)$ are denoted by

$$
N_{L / K}:=j_{*}: \mathscr{M}(L) \rightarrow \mathscr{M}(K), \quad \text { and } \quad \operatorname{Res}_{L / K}:=j^{*}: \mathscr{M}(K) \rightarrow \mathscr{M}(L) .
$$

The category of Mackey functors over $k$ forms an abelian category with the following tensor product:

Definition $2.2(c f .[8])$. For Mackey functors $\mathscr{M}$ and $\mathscr{N}$, their Mackey product $\mathscr{M} \otimes \mathscr{N}$ is defined as follows: For any finite field extension $k^{\prime} / k$,

$$
(\mathscr{M} \otimes \mathscr{N})\left(k^{\prime}\right):=\left(\bigoplus_{K / k^{\prime}: \text { finite }} \mathscr{M}(K) \otimes_{\mathbb{Z}} \mathscr{N}(K)\right) /(\mathbf{P F}),
$$

where (PF) stands for the subgroup generated by elements of the following form:

(PF) For finite field extensions $k^{\prime} \subset K \subset L$,

$$
\begin{array}{ll}
N_{L / K}(x) \otimes y-x \otimes \operatorname{Res}_{L / K}(y) & \text { for } x \in \mathscr{M}(L) \text { and } y \in \mathscr{N}(K), \text { and } \\
x \otimes \operatorname{Res}_{L / K}(y)-N_{L / K}(x) \otimes y & \text { for } x \in \mathscr{M}(K) \text { and } y \in \mathscr{N}(L) .
\end{array}
$$


For the Mackey product $\mathscr{M} \otimes \mathscr{N}$, we write $\{x, y\}_{K / k^{\prime}}$ for the image of $x \otimes y \in \mathscr{M}(K) \otimes_{\mathbb{Z}} \mathscr{N}(K)$ in the product $(\mathscr{M} \otimes \mathscr{N})\left(k^{\prime}\right)$. For any finite field extension $k^{\prime} / k$, the push-forward

$$
N_{k^{\prime} / k}=j_{*}:(\mathscr{M} \otimes \mathscr{N})\left(k^{\prime}\right) \longrightarrow(\mathscr{M} \otimes \mathscr{N})(k)
$$

is given by $N_{k^{\prime} / k}\left(\{x, y\}_{K / k^{\prime}}\right)=\{x, y\}_{K / k}$. For each $m \in \mathbb{Z}_{\geq 1}$, we define a Mackey functor $\mathscr{M} / m$ by

$$
(\mathscr{M} / m)(K):=\mathscr{M}(K) / m
$$

for any finite extension $K / k$. We have

$$
(\mathscr{M} / m \otimes \mathscr{N} / m)(k) \simeq(\mathscr{M} \otimes \mathscr{N})(k) / m \quad(=((\mathscr{M} \otimes \mathscr{N}) / m)(k) \text { in the sense of }(\underline{3})) .
$$

Every $G_{k}$-module $M$ defines a Mackey functor defined by the fixed sub module $M(K):=$ $M^{\mathrm{Gal}(\bar{k} / K))}$ denoted by $M$. Conversely, assume a Mackey functor $\mathscr{M}$ satisfies the Galois descent, meaning that, for every finite Galois extensions $L / K$, the restriction

$$
\operatorname{Res}_{L / K}: \mathscr{M}(K) \stackrel{\simeq}{\rightarrow} \mathscr{M}(L)^{\mathrm{Gal}(L / K)}
$$

is an isomorphism. This Mackey functor $\mathscr{M}$ gives a $G_{k}$-module $\mathscr{M}=\lim _{\longrightarrow / k} \mathscr{M}(K)(c f$. [13], Chap. 1, Sect. 5, Ex. 1) denoted again by $\mathscr{M}$. For any $m \in \mathbb{Z}_{\geq 1}$, the connecting homomorphism associated to the short exact sequence $0 \rightarrow \mathscr{M}[m] \rightarrow \mathscr{M} \stackrel{m}{\rightarrow} \mathscr{M} \rightarrow 0$ as $G_{k}$-modules gives

$$
\delta_{\mathscr{M}}: \mathscr{M}(K) / m \hookrightarrow H^{1}(K, \mathscr{M}[m])
$$

which is often called the Kummer map.

Definition 2.3 ( $c f$. [19], Prop. 1.5). For Mackey functors $\mathscr{M}$ and $\mathscr{N}$ with Galois descent, the Galois symbol map

$$
s_{m}^{M}:(\mathscr{M} \otimes \mathscr{N})(k) / m \rightarrow H^{2}(k, \mathscr{M}[m] \otimes \mathscr{N}[m])
$$

is defined by the cup product and the corestriction as follows:

$$
s_{m}^{M}\left(\{x, y\}_{K / k}\right)=\operatorname{Cor}_{K / k}\left(\delta_{\mathscr{M}}(x) \cup \delta_{\mathscr{N}}(y)\right) .
$$

\section{Somekawa $K$-group}

For two semi-abelian varieties $G_{1}$ and $G_{2}$ over $k$, the $G_{k}$-modules $G_{1}(\bar{k})$ and $G_{2}(\bar{k})$ define a Mackey functors with Galois descent which we denote also by $G_{1}$ and $G_{2}$. The Somekawa $K$-group $K\left(k ; G_{1}, G_{2}\right)$ is a quotient of $\left(G_{1} \otimes G_{2}\right)(k)$ (for the definition, see [19], [14]). The Galois symbol map $s_{m}^{M}:\left(G_{1} \otimes G_{2}\right)(k) / m \rightarrow H^{2}\left(k, G_{1}[m] \otimes G_{2}[m]\right)$ (Def. 2.3) factors through $K\left(k ; G_{1}, G_{2}\right)$ and the induced map

$$
s_{m}: K\left(k ; G_{1}, G_{2}\right) / m \rightarrow H^{2}\left(k, G_{1}[m] \otimes G_{2}[m]\right)
$$

is also called the Galois symbol map. Somekawa presented a "conjecture" in which the map $s_{m}$ is injective (for arbitrary field). For the case $G_{1}=G_{2}=\mathbb{G}_{m}$, as $K\left(k ; \mathbb{G}_{m}, \mathbb{G}_{m}\right) \simeq K_{2}^{M}(k)$ the conjecture holds by the Merkurjev-Suslin theorem ([12]). Although it holds in some special cases ([23], [24], and [14]), Spieß and Yamazaki disproved this for some tori ([20], Prop. 7). 


\section{Class field theory}

Following [1], [15], we recall the class field theory for a curve over $k$. Let $X$ be a projective smooth and geometrically connected curve over $k$. Define

- $X_{0}$ : the set of closed points in $X$,

- $k(X)$ : the function field of $X$,

- $k(x)$ : the residue field at $x \in X_{0}$, and

- $k(X)_{x}$ : the completion of $k(X)$ at $x \in X_{0}$.

We define

$$
S K_{1}(X):=\operatorname{Coker}\left(\partial: K_{2}(k(X)) \rightarrow \bigoplus_{x \in X_{0}} k(x)^{\times}\right),
$$

where the map $\partial$ is given by the direct sum of the boundary map $K_{2}\left(k(X)_{x}\right) \rightarrow K_{1}(k(x))=k(x)^{\times}$ for $x \in X_{0}$. Note that the residue field $k(x)$ is a finite extension field of $k$ so that $k(x)$ is also a $p$-adic field. The reciprocity maps $k(x)^{\times} \rightarrow \pi_{1}^{\mathrm{ab}}(x)$ of the local class field theory of $k(x)$ for $x \in X_{0}$ induce the reciprocity map

$$
\rho: S K_{1}(X) \rightarrow \pi_{1}^{\mathrm{ab}}(X) .
$$

The map $\rho$ is compatible with the reciprocity map $\rho_{k}: k^{\times} \rightarrow G_{k}^{\mathrm{ab}}=\operatorname{Gal}\left(k^{\mathrm{ab}} / k\right)=\pi_{1}^{\mathrm{ab}}(\operatorname{Spec}(k))$ of the base field $k$ as in the following commutative diagram:

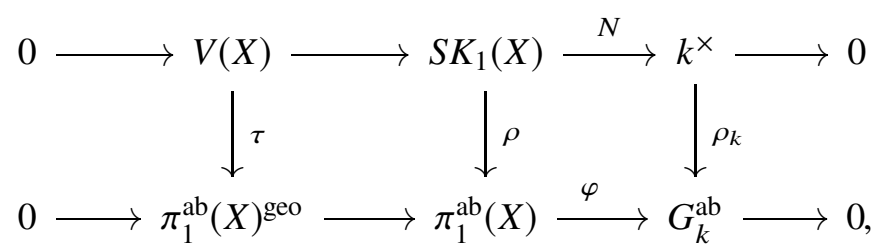

where the map $\varphi$ is induced from the structure map $X \rightarrow \operatorname{Spec}(k), N$ is induced from the norm maps $k(x)^{\times} \rightarrow k^{\times}$for each $x \in X_{0}$, and the groups $V(X)$ and $\pi_{1}^{\mathrm{ab}}(X)^{\text {geo }}$ are defined by the exactness. The main theorem of the class field theory for $X$ is the following:

Theorem 2.4 ([1], [15]). (i) $\pi_{1}^{\mathrm{ab}}(X) / \overline{\operatorname{Im}(\rho)} \simeq \widehat{\mathbb{Z}}^{\oplus r}$ for some $r \geq 0$, where $\overline{\operatorname{Im}(\rho)}$ is the topological closure of the image $\operatorname{Im}(\rho)$ in $\pi_{1}^{\mathrm{ab}}(X)$.

(ii) $\operatorname{Ker}(\rho)=S K_{1}(X)_{\mathrm{div}}$, where $S K_{1}(X)_{\mathrm{div}}$ is the maximal divisible subgroup of $S K_{1}(X)$.

(iii) $\operatorname{Ker}(\tau)=V(X)_{\mathrm{div}}$, where $V(X)_{\mathrm{div}}$ is the maximal divisible subgroup of $V(X)$.

(iv) $\operatorname{Im}(\tau)$ is finite.

Note that the invariant $r$ above is determined by the special fiber of the Néron model of the Jacobian variety $J=\operatorname{Jac}(X)$. In particular, we have $r=0$ if $X$ has good reduction. From the above theorem, the group $V(X)$ has a decomposition

$$
V(X)=V(X)_{\mathrm{div}} \oplus V(X)_{\mathrm{fin}},
$$


where the reduced part $V(X)_{\text {fin }}$ is a finite subgroup.

From now on, we assume that $X(k) \neq \emptyset$. The geometric fundamental group $\pi_{1}^{\mathrm{ab}}(X)^{\text {geo }}$ is written by the Tate module $T(J)=\lim _{m} J[m]$ of the Jacobian variety $J=\operatorname{Jac}(X)$ as

$$
\pi_{1}^{\mathrm{ab}}(X)^{\mathrm{geo}} \simeq T(J)_{G_{k}},
$$

where $T(J)_{G_{k}}$ is the $G_{k}$-coinvariant quotient of $T(J)$ ([16], Chap. II, Lem. 3.2). On the other hand, the group $V(X)$ is written by the Somekawa $K$-group as

$$
V(X) \simeq K\left(k ; \mathbb{G}_{m}, J\right)
$$

([19], Thm. 2.1, [14], Rem. 2.4.2 (c)). The reciprocity map $\tau$ in the diagram (8) coincides with the Galois symbol map associated with $\mathbb{G}_{m}$ and $J$ as in the following commutative diagram:

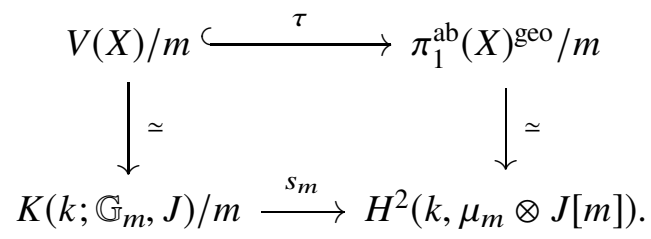

Here, the right vertical map is induced from $H^{2}\left(k, \mu_{m} \otimes J[m]\right) \simeq J[m]_{G_{k}}$ which is given by the local Tate duality theorem. From the construction, the composition $V(X) / m \simeq K\left(k ; \mathbb{G}_{m}, J\right) / m \stackrel{s_{m}}{\rightarrow}$ $H^{2}\left(k, \mu_{m} \otimes J[m]\right)$ is compatible with the map $V(X) \rightarrow H^{2}\left(k, \mu_{m} \otimes J[m]\right)$ given by Bloch ([1], Thm. 1.14). From the diagram above, we have $V(X) / m \simeq \operatorname{Im}\left(s_{m}\right)$. For the prime to $p$-part of $V(X)$, we have the following proposition:

Proposition 2.5 ([1], Prop. 2.29). Assume that $X$ has good reduction and $X(k) \neq \emptyset$. Then, for any $m \in \mathbb{Z}_{\geq 1}$ prime to $p$, we have $V(X) / m \simeq \bar{J}(\mathbb{F}) / m$, where $\bar{J}$ is the reduction of $J$.

Note that if $X$ has good reduction, then the Jacobian variety $J=\operatorname{Jac}(X)$ has also good reduction. But, the converse does not hold.

\section{Prime to $p$-part}

We extend Proposition 2.5 using the Somekawa $K$-group to an abelian variety following Bloch's proof of the above proposition essentially.

Proposition 2.6. Let $A$ be an abelian variety over $k$ which has good reduction. For any $m \in \mathbb{Z}_{\geq 1}$ prime to $p$, we have $K\left(k ; \mathbb{G}_{m}, A\right) / m \simeq \bar{A}(\mathbb{F}) / m$.

Proof. The kernel of the reduction $\pi: A(k) \rightarrow \bar{A}(\mathbb{F})$ is isomorphic to the formal group $\widehat{A}\left(\mathfrak{m}_{k}\right)=$ : $\widehat{A}(k)$ which has no prime to $p$ torsion ([6], Prop. C.2.5, Thm. C.2.6). The reduction map induces $A(k) / m \simeq \bar{A}(\mathbb{F}) / m$ for each $m$ prime to $p$. 
First, we define $\psi: A(k) / m \rightarrow K\left(k ; \mathbb{G}_{m}, A\right) / m$ by

$$
\psi(x)=\{\pi, x\}_{k / k},
$$

where $\pi$ is a uniformizer of $k$. The map does not depend on the choice of $\pi$. In fact, for any $u \in O_{k}^{\times}$, by taking $\xi \in[m]^{-1}(x), K=k(\xi) / k$ is unramified. By local class field theory, there exists $\mu \in K^{\times}$such that $N_{K / k} \mu=u$. We have $\{u, x\}_{k / k}=\left\{N_{K / k} \mu, x\right\}_{k / k}=\left\{\mu, \operatorname{Res}_{K / k} x\right\}_{K / k}=$ $\{\mu, m \xi\}_{k / k}=0$ in $K\left(k ; \mathbb{G}_{m}, A\right) / m$.

Claim. $\psi$ is surjective.

Proof. Take an element of the form $\left\{\mu \varpi^{n}, \xi\right\}_{K / k}$ in $K\left(k ; \mathbb{G}_{m}, A\right) / m$, where $\varpi$ is the uniformizer in $K$, and $\mu \in O_{K}^{\times}$. As above, we have $\{\mu, \xi\}_{K / k}=0$ by considering the unramified extension $K\left([m]^{-1} \xi\right) / K$. It is enough to show that the element $\{\varpi, \xi\}_{K / k}$ is generated by the elements of the form $\{\pi, x\}_{k / k}$.

Let $k^{\prime}$ be the maximal unramified subextension of $K / k . \pi^{\prime}=\operatorname{Res}_{k^{\prime} / k} \pi$ is also a uniformizer of $k^{\prime}$. The extension $K / k^{\prime}$ is totally ramified so that we may take $\varpi$ as $N_{K / k^{\prime}} \varpi=\pi^{\prime}$. Since the restriction $\operatorname{Res}_{K / k^{\prime}}: A\left(k^{\prime}\right) / m \stackrel{\simeq}{\rightarrow} A(K) / m$ is bijective, there exists $x^{\prime} \in A\left(k^{\prime}\right) / m$ such that $\operatorname{Res}_{K / k^{\prime}} x^{\prime}=\xi$. Therefore

$$
\begin{aligned}
\{\varpi, \xi\}_{K / k} & =\left\{\varpi, \operatorname{Res}_{K / k^{\prime}} x^{\prime}\right\}_{K / k} \\
& =\left\{N_{K / k^{\prime}} \varpi, x^{\prime}\right\}_{k^{\prime} / k} \quad \text { (by the projection formula) } \\
& =\left\{\operatorname{Res}_{k^{\prime} / k} \pi, x^{\prime}\right\}_{k^{\prime} / k} \\
& =\left\{\pi, N_{k^{\prime} / k} x^{\prime}\right\}_{k / k} \quad \text { (by the projection formula). }
\end{aligned}
$$

From these equalities, the map $\psi$ is surjective.

Next, we show the map $\psi$ is bijective by showing the following claim.

Claim. For any $m$ prime to $p$, we have $\# K\left(k ; \mathbb{G}_{m}, A\right) / m \geq \# \bar{A}(\mathbb{F}) / m$.

Proof. The direct limit of the Galois symbol map

$$
\lim _{\longleftarrow} s_{m}: K\left(k ; \mathbb{G}_{m}, A\right) \rightarrow \lim _{m \geq 1} H^{2}\left(k, \mu_{m} \otimes A[m]\right) \simeq T(A)_{G_{k}}
$$

is known to be surjective ([4], Thm. A,1), where $T(A)_{G_{k}}$ is the $G_{k}$-coinvariant quotient of the Tate module $T(A)=\lim _{m} A[m]$ of $A$ and the latter isomorphism follows from the local Tate duality theorem. Write $T(A)=T_{p}(A) \times T^{\prime}(A)$, where $T^{\prime}(A)=\lim _{(m, p)=1} A[m]$. As $A$ has good reduction, the inertia subgroup $I \subset G_{k}$ acts trivially on $T^{\prime}(A)$ so that $T^{\prime}(A)_{G_{k}} \simeq T^{\prime}(\bar{A})_{G_{F}}$, where $T^{\prime}(\bar{A})=\lim _{(m, p)=1} \bar{A}[m]$. The Weil conjecture for abelian varieties implies that $T^{\prime}(\bar{A})_{G_{\mathbb{F}}}$ is isomorphic to the prime to $p$-part of the torsion subgroup of $\bar{A}(\mathbb{F})$ (cf. [1], Prop. 2.4; [9], Thm. 1 (ter)). For any $m$ prime to $p$, we have

$$
\# K\left(k ; \mathbb{G}_{m}, A\right) / m \geq \# T(A)_{G_{k}} / m=\# T^{\prime}(\bar{A})_{G_{\mathbb{F}}} / m=\# \bar{A}(\mathbb{F}) / m .
$$


From the above claims, the surjective homomorphism

$$
\bar{A}(\mathbb{F}) / m \simeq A(k) / m \stackrel{\psi}{\rightarrow} K\left(k ; \mathbb{G}_{m}, A\right) / m
$$

is bijective by comparing the cardinality.

\section{Kummer map}

In this section, let $A$ be an abelian variety of dimension $g$ over $k$ assuming

(Ord) $A$ has good ordinary reduction, in the sense that $A$ has good reduction and its reduction $\bar{A}$ has ordinary reduction, and

(Rat) $A[p] \subset A(k)$.

Let $k^{\text {ur }}$ be the completion of the maximal unramified extension of $k$. The kernel of the reduction is

$$
A_{1}\left(k^{\mathrm{ur}}\right)=\operatorname{Ker}\left(\pi: A\left(k^{\mathrm{ur}}\right) \rightarrow \bar{A}(\mathbb{F})\right) \simeq \widehat{A}\left(\mathrm{~m}_{k^{\mathrm{ur}}}\right)=: \widehat{A}\left(k^{\mathrm{ur}}\right)
$$

(cf. [6], Thm. C.2.6). It is known that we have $\widehat{A} \times_{O_{k}} \operatorname{Spf}\left(O_{k^{\text {ur }}}\right) \simeq\left(\widehat{\mathbb{G}}_{m}\right)^{\oplus g}$, where $\widehat{\mathbb{G}}_{m}$ is the multiplicative group ([11], Lem. 4.26, Lem. 4.27). Since we have $A[p] \subset A(k), A_{1}[p] \subset A_{1}(k)$ and hence we obtain isomorphisms

$$
A_{1}[p]=A_{1}\left(k^{\mathrm{ur}}\right)[p] \simeq \widehat{A}\left(k^{\mathrm{ur}}\right)[p] \simeq\left(\left(\widehat{\mathbb{G}}_{m}\right)\left(k^{\mathrm{ur}}\right)[p]\right)^{\oplus g} \simeq\left(\mu_{p}\right)^{\oplus g} .
$$

Now we choose an isomorphism

$$
A[p] \stackrel{\simeq}{\rightarrow}\left(\mu_{p}\right)^{\oplus 2 g}
$$

of (trivial) Galois modules which makes the following diagram commutative:

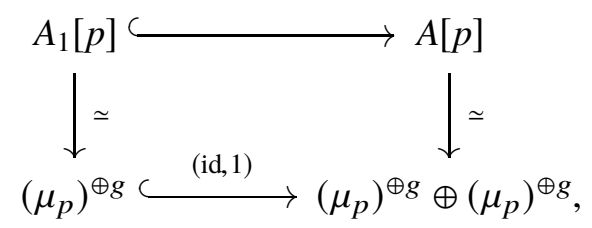

where the left vertical map is given in (11), and the bottom horizontal map is defined by

$$
\left(\mu_{p}\right)^{\oplus g} \rightarrow\left(\mu_{p}\right)^{\oplus 2 g} ;\left(x_{1}, \ldots, x_{g}\right) \mapsto\left(x_{1}, \ldots, x_{g}, 1, \ldots, 1\right) .
$$

In the following, the Kummer map on $\mathbb{G}_{m}$ gives the isomorphism $\delta_{\mathbb{G}_{m}}: K^{\times} / p \stackrel{\simeq}{\rightarrow} H^{1}\left(K, \mu_{p}\right)$ for an extension $K / k$ and we identify these groups. The fixed isomorphism (12) induces an isomorphism (*) below

$$
\delta_{A}^{K}: A(K) / p \stackrel{\delta_{A}}{\longrightarrow} H^{1}(K, A[p]) \stackrel{(\bullet)}{\simeq} H^{1}\left(K, \mu_{p}\right)^{\oplus 2 g}=\left(K^{\times} / p\right)^{\oplus 2 g} .
$$


Proposition 3.1. For any finite extension $K / k$, we have the following:

(i) The image of the Kummer map $\delta_{A}^{K}$ equals to

$$
\left(\bar{U}_{K}\right)^{\oplus g} \oplus \operatorname{Ker}\left(K^{\times} / p \stackrel{j}{\rightarrow}\left(K^{\mathrm{ur}}\right)^{\times} / p\right)^{\oplus g}
$$

where $\bar{U}_{K}:=\operatorname{Im}\left(U_{K} \rightarrow K^{\times} / p\right), K^{\mathrm{ur}}$ is the completion of the maximal unramified extension of $K$, and $j$ is the map induced from the inclusion $K^{\times} \hookrightarrow\left(K^{\mathrm{ur}}\right)^{\times}$.

(ii) The image of the composition $A_{1}(K) / p \rightarrow A(K) / p \stackrel{\delta_{A}^{K}}{\rightarrow}\left(K^{\times} / p\right)^{\oplus 2 g}$ coincides with $\left(\bar{U}_{K}\right)^{\oplus g}$. In particular, $\widehat{A}(K) / p \simeq A_{1}(K) / p \simeq\left(\bar{U}_{K}\right)^{\oplus g}$.

In the following, we fix a finite extension $K / k$ and prove the above proposition. First, we show the following lemma on $\delta_{A_{1}}^{K^{\mathrm{ur}}}: A_{1}\left(K^{\mathrm{ur}}\right) / p \hookrightarrow H^{1}\left(K^{\mathrm{ur}}, A_{1}[p]\right) \stackrel{(\vartheta)}{\simeq}\left(\left(K^{\mathrm{ur}}\right)^{\times} / p\right)^{\oplus g}$, where the isomorphism $(\diamond)$ is given by (11).

Lemma 3.2. (i) $\operatorname{Im}\left(\delta_{A_{1}}^{K^{\mathrm{ur}}}\right) \subset\left(\bar{U}_{K^{\mathrm{ur}}}\right)^{\oplus g}$.

(ii) $\operatorname{Im}\left(\delta_{A}^{K^{\mathrm{ur}}}\right) \subset\left(\bar{U}_{K^{\mathrm{ur}}}\right)^{\oplus g}$.

Proof. (i) Recall that we have $A_{1}\left(K^{\text {ur }}\right) \simeq \widehat{A}\left(K^{\text {ur }}\right) \simeq\left(\widehat{\mathbb{G}}_{m}\left(K^{\text {ur }}\right)\right)^{\oplus g}$ as noted above. The isomorphism (11) gives the following commutative diagram:

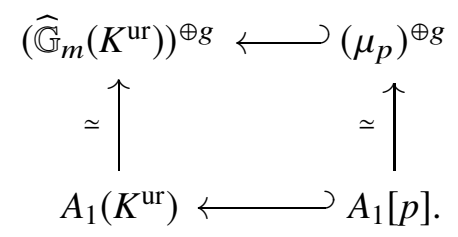

The above diagram makes the square ( $)$ commutative in the next diagram.

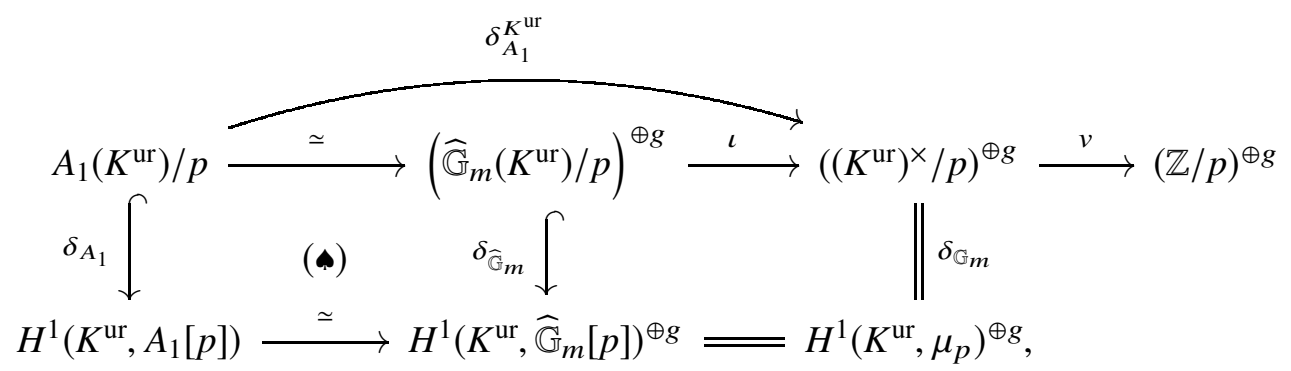

where $\iota$ is induced from $\widehat{\mathbb{G}}_{m}\left(K^{\text {ur }}\right)=U_{K^{u r}}^{1} \hookrightarrow\left(K^{\text {ur }}\right)^{\times}$and $v$ is the valuation map. Since $\widehat{\mathbb{G}}_{m}\left(K^{\text {ur }}\right)=$ $U_{K^{\text {ur }}}^{1} \subset O_{K^{\text {ur }}}^{\times}, v \circ \iota=0$ in the diagrm (14). Hence, the claim $\operatorname{Im}\left(\delta_{A_{1}}^{K^{\mathrm{ur}}}\right) \subset\left(\bar{U}_{K^{\mathrm{ur}}}\right)^{\oplus g}=\operatorname{Ker}(v:$ $\left.\left(K^{\mathrm{ur}} / p\right)^{\oplus g} \rightarrow(\mathbb{Z} / p)^{\oplus g}\right)$ holds. 
(ii) Consider the following short exact sequence

$$
A_{1}\left(K^{\mathrm{ur}}\right) / p \rightarrow A\left(K^{\mathrm{ur}}\right) / p \rightarrow \bar{A}\left(\mathbb{F}_{K^{\mathrm{ur}}}\right) / p \rightarrow 0 .
$$

Since the residue field $\mathbb{F}_{K^{\text {ur }}}=\overline{\mathbb{F}}_{K}$ is algebraically closed, $\bar{A}\left(\mathbb{F}_{K^{\text {ur }}}\right) / p=\bar{A}\left(\overline{\mathbb{F}}_{K}\right) / p=0$ and hence the natural map $A_{1}\left(K^{\mathrm{ur}}\right) / p \rightarrow A\left(K^{\mathrm{ur}}\right) / p$ is surjective. This map gives the commutative diagram below:

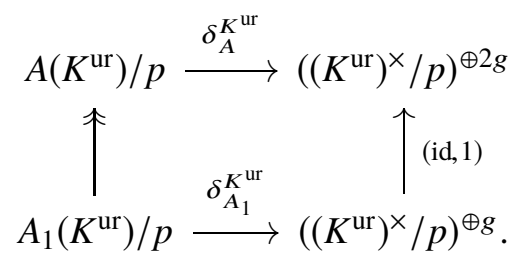

From this diagram, the image of $\delta_{A}^{K^{\text {ur }}}$ is contained in $\left(\bar{U}_{K^{\text {ur }}}\right)^{\oplus g}$ as claimed.

Next, we study the image of

$$
\delta_{A_{1}}^{K}: A_{1}(K) / p \hookrightarrow H^{1}\left(K, A_{1}[p]\right) \simeq H^{1}\left(K, \mu_{p}\right)^{\oplus g}=\left(K^{\times} / p\right)^{\oplus g},
$$

Lemma 3.3. $\operatorname{Im}\left(\delta_{A_{1}}^{K}\right)=\left(\bar{U}_{K}\right)^{\oplus g}$.

Proof. First, we show the following claim:

Claim. $\operatorname{Im}\left(\delta_{A_{1}}^{K}\right) \subset\left(\bar{U}_{K}\right)^{\oplus g}$.

Proof. Consider the following commutative diagram:

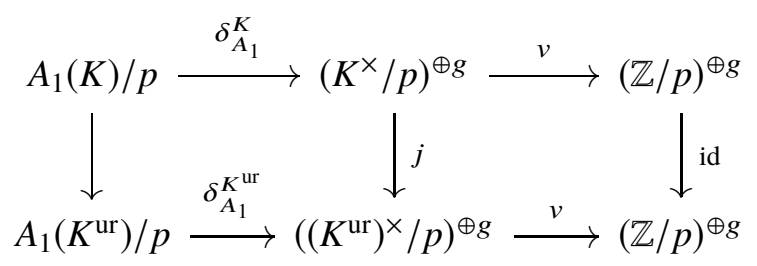

From Lemma 3.2 (i), the composition $v \circ \delta_{A_{1}}^{K^{\text {ur }}}=0$ in the above diagram. The composition $v \circ \delta_{A_{1}}^{K}=0$ in the top sequence and hence $\operatorname{Im}\left(\delta_{A_{1}}\right) \subset\left(\bar{U}_{K}\right)^{\oplus g}$.

Next, we compare the orders of both of $\operatorname{Im}\left(\delta_{A_{1}}^{K}\right)$ and $\left(\bar{U}_{K}\right)^{\oplus g}$. As $\bar{U}_{K}=\operatorname{Ker}\left(v: K^{\times} / p \rightarrow \mathbb{Z} / p\right)$, the last claim indicates

$$
\# A_{1}(K) / p \leq \#\left(\bar{U}_{K}\right)^{\oplus g}=p^{g\left(\left[K: \mathbb{Q}_{p}\right]+1\right)} .
$$

On the other hand, Mattuck's theorem ([10]) and the assumption $A[p] \subset A(K)$ say

$$
A(K) / p \simeq(\mathbb{Z} / p)^{\oplus g\left(\left[K: \mathbb{Q}_{p}\right]+2\right)} .
$$


The short exact sequence

$$
A_{1}(K) / p \rightarrow A(K) / p \rightarrow \bar{A}\left(\mathbb{F}_{K}\right) / p \rightarrow 0
$$

and $\# \bar{A}\left(\mathbb{F}_{K}\right) / p=\# \bar{A}\left(\mathbb{F}_{K}\right)[p]=p^{g}$ as $\bar{A}$ has ordinary reduction, we obtain the inequality

$$
\# A_{1}(K) / p \geq p^{g\left(\left[K: \mathbb{Q}_{p}\right]+1\right)} .
$$

Therefore, the map $\delta_{A_{1}}^{K}: A_{1}(K) / p \stackrel{\simeq}{\rightarrow}\left(\bar{U}_{K}\right)^{\oplus g}$ is bijective.

Proof of Prop. 3.1

Claim. $\operatorname{Im}\left(\delta_{A}^{K}\right) \subset\left(\bar{U}_{K}\right)^{\oplus g} \oplus \operatorname{Ker}\left(j: K^{\times} / p \rightarrow\left(K^{\mathrm{ur}}\right)^{\times} / p\right)^{\oplus g}$.

Proof. Consider the following commutative diagram:

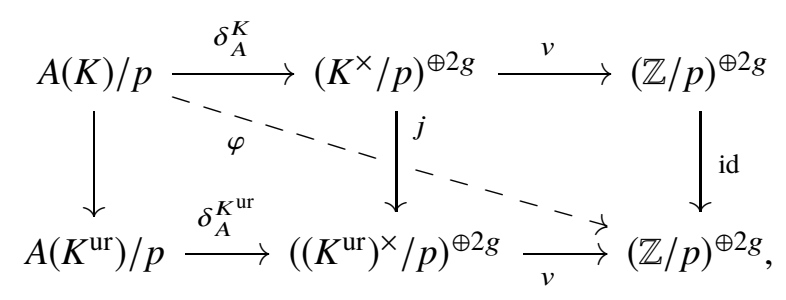

From Lemma 3.2 (ii), the image of the composition $j \circ \delta_{A}^{K}$ is contained in $\left(\bar{U}_{K^{\mathrm{ur}}}\right)^{\oplus g}$. In particular, the image of $\varphi$ the dotted arrow in the above diagram is 0 so that we obtain $\operatorname{Im}\left(\delta_{A}^{K}\right) \subset$ $\left(\bar{U}_{K}\right)^{\oplus g} \oplus \operatorname{Ker}(j)^{\oplus g}$.

(i) From Mattuck's theorem ([10]) and the assumption $A[p] \subset A(k)$,

$$
A(K) / p \simeq(\mathbb{Z} / p)^{\oplus g\left(\left[K: \mathbb{Q}_{p}\right]+2\right)} .
$$

On the other hand,

$$
\operatorname{Ker}\left(K^{\times} / p \rightarrow\left(K^{\mathrm{ur}}\right)^{\times} / p\right) \simeq \operatorname{Ker}\left(H^{1}\left(K, \mu_{p}\right) \rightarrow H^{1}\left(K^{\mathrm{ur}}, \mu_{p}\right)\right) \simeq H^{1}\left(K^{\mathrm{ur}} / K, \mu_{p}\right) \simeq \mathbb{Z} / p,
$$

and hence

$$
\#\left(\bar{U}_{K} \oplus \operatorname{Ker}\left(K^{\times} / p \rightarrow\left(K^{\mathrm{ur}}\right)^{\times} / p\right)\right)^{\oplus g}=\#\left(K^{\times} / p\right)^{\oplus g}=p^{g\left(\left[K: \mathbb{Q}_{p}\right]+2\right)} .
$$

By counting the cardinality, we obtain the equality $\operatorname{Im}\left(\delta_{A}^{K}\right)=\left(\bar{U}_{K}\right)^{\oplus g} \oplus \operatorname{Ker}\left(K^{\times} / p \rightarrow\left(K^{\mathrm{ur}}\right)^{\times} / p\right)^{\oplus g}$.

(ii) From Lemma 3.3 and the commutative diagram below give the assertion.

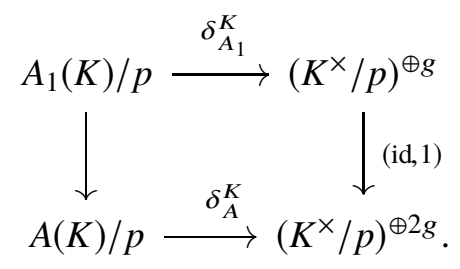


Now we define the sub Mackey functors $\mathscr{U}, \mathscr{V} \subset \mathbb{G}_{m} / p$ by

$$
\mathscr{U}(K):=\bar{U}_{K}=\operatorname{Im}\left(U_{K} \rightarrow K^{\times} / p\right), \quad \mathscr{V}(K):=\operatorname{Ker}\left(j: K^{\times} / p \rightarrow\left(K^{\mathrm{ur}}\right)^{\times} / p\right),
$$

for any finite extension $K / k$. Note that, we have

$$
\mathscr{V}(K)=\operatorname{Im}\left(U_{K}^{p e_{0}(K)} \rightarrow K^{\times} / p\right),
$$

where $e_{0}(K)=e_{K} /(p-1)$ and $e_{K}$ is the ramification index of $K / \mathbb{Q}_{p}$ (cf. [21], Rem. 3.2). In fact, both of the subgroups of $K^{\times} / p$ are annihilators of $\bar{U}_{K}$ in the Hilbert symbol. By the fixed isomorphism (12), the following diagram is commutative

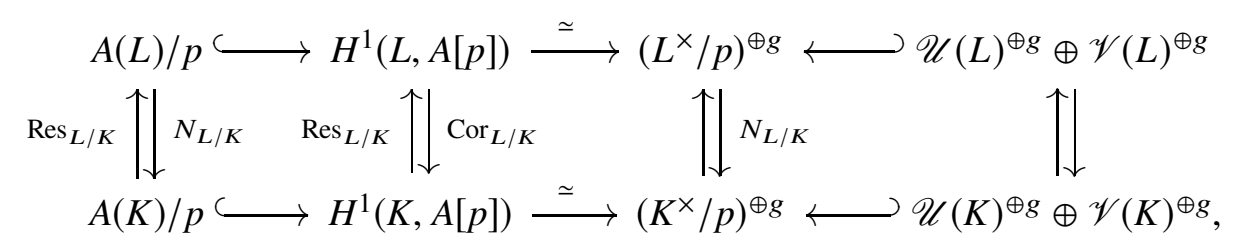

for any finite extensions $L / K / k$. We obtain the following isomorphisms of Mackey functors:

Corollary 3.4. There are isomorphisms

$$
A / p \simeq \mathscr{U}^{\oplus g} \oplus \mathscr{V}^{\oplus g}, \quad \text { and } \quad \widehat{A} / p \simeq \mathscr{U}^{\oplus g}
$$

of Mackey functors.

\section{Galois symbol map}

Let $A$ be an abelian variety over $k$ which has good reduction. The formal group $\widehat{A}$ defines a Mackey functor by the associated group $\widehat{A}(K):=\widehat{A}\left(\mathrm{~m}_{K}\right)$ for a finite extension $K / k$. Note that we have $\widehat{A}(K) \simeq A_{1}(K)$ ([HS], Thm. C.2.6). There is a short exact sequence of Mackey functors

$$
0 \rightarrow \widehat{A} \rightarrow A \rightarrow \overline{\mathscr{A}} \rightarrow 0
$$

where $\overline{\mathscr{A}}=A / \widehat{A}$ is defined by the exactness (in the abelian category of Mackey functors). The Mackey functor $\overline{\mathscr{A}}$ has the following description (cf. [14], Sect. 3, (3.3)): For a finite extension $K / k$ with residue field $\mathbb{F}_{K}$,

$$
\overline{\mathscr{A}}(K) \simeq \bar{A}\left(\mathbb{F}_{K}\right) .
$$

For finite extensions $L / K / k$ with ramification index $e(L / K)$, the restriction $\operatorname{Res}_{L / K}: \overline{\mathscr{A}}(K) \rightarrow$ $\overline{\mathscr{A}}(L)$ and the norm map $N_{L / K}: \overline{\mathscr{A}}(L) \rightarrow \overline{\mathscr{A}}(K)$ can be identified with the restriction $\bar{A}\left(\mathbb{F}_{L}\right) \rightarrow$ $\bar{A}\left(\mathbb{F}_{K}\right)$ and $e(L / K) N_{\mathbb{F}_{L} / \mathbb{F}_{K}}: \bar{A}\left(\mathbb{F}_{L}\right) \rightarrow \bar{A}\left(\mathbb{F}_{K}\right)$ respectively.

For each $n \geq 1$, we denote by

$$
\widehat{\mathscr{M}}_{n}:=\left(\mathbb{G}_{m} / p^{n} \otimes \widehat{A} / p^{n}\right)(k), \quad \text { and } \quad \overline{\mathscr{M}}_{n}:=\left(\mathbb{G}_{m} / p^{n} \otimes \overline{\mathscr{A}} / p^{n}\right)(k) .
$$


By applying $\mathbb{G}_{m} / p^{n} \otimes-$ (which is right exact) to the sequence (17), we have

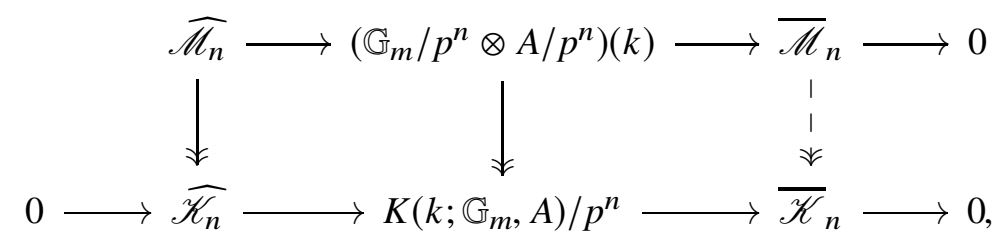

where $\widehat{\mathscr{K}}_{n}$ is the image of the composition $\widehat{\mathscr{M}}_{n} \rightarrow\left(\mathbb{G}_{m} / p^{n} \otimes A / p^{n}\right)(k) \rightarrow K\left(k ; \mathbb{G}_{m}, A\right) / p^{n}$ and $\overline{\mathscr{K}}_{n}$ is defined by the exactness of the lower sequence.

\section{Special fiber}

Lemma 4.1. Let $A$ be an abelian variety over $k$ with good reduction. Then, we have

$$
\overline{\mathscr{M}}_{n}=\left(\mathbb{G}_{m} / p^{n} \otimes \overline{\mathscr{A}} / p^{n}\right)(k) \simeq \bar{A}(\mathbb{F}) / p^{n}
$$

for any $n \in \mathbb{Z}_{\geq 1}$.

Proof. First, we define a Mackey functor $\mathscr{Z}$ by $\mathscr{Z}(K)=\mathbb{Z}$ for each finite extension $K / k$ and, for a finite extensions $L / K / k$, the norm $N_{L / K}: \mathscr{Z}(L) \rightarrow \mathscr{Z}(K)$ is multiplication by the residue degree $f(L / K)$ of $L / K$ and the restriction $\operatorname{Res}_{L / K}: \mathscr{Z}(K) \rightarrow \mathscr{Z}(L)$ is multiplication by the ramification index $e(L / K)$ of $L / K$. The valuations give a morphism $v: \mathbb{G}_{m} \rightarrow \mathscr{Z}$ of Mackey functors defined by the valuation map

$$
v: \mathbb{G}_{m}(K)=K^{\times} \rightarrow \mathscr{Z}(K)=\mathbb{Z},
$$

of $K$, for any finite extension $K / k$. As in (16), we define a Mackey functor $\mathscr{U}_{n}$ by

$$
\mathscr{U}_{n}(K):=\operatorname{Im}\left(U_{K} \rightarrow K^{\times} / p^{n}\right)
$$

which fits into the short exact sequence

$$
0 \rightarrow \mathscr{U}_{n} \rightarrow \mathbb{G}_{m} / p^{n} \stackrel{v}{\rightarrow} \mathscr{Z} / p^{n} \rightarrow 0 .
$$

From the right exactness of $-\otimes \overline{\mathscr{A}} / p^{n}$ for $\overline{\mathscr{A}}=A / \widehat{A}(c f .(18))$, we have

$$
\mathscr{U}_{n} \otimes \overline{\mathscr{A}} / p^{n} \rightarrow \mathbb{G}_{m} / p^{n} \otimes \overline{\mathscr{A}} / p^{n} \rightarrow \mathscr{Z} / p^{n} \otimes \overline{\mathscr{A}} / p^{n} \rightarrow 0 .
$$

Claim. $\left(\mathscr{Z} / p^{n} \otimes \overline{\mathscr{A}} / p^{n}\right)(k) \simeq \bar{A}(\mathbb{F}) / p^{n}$

Proof. By identifying $\overline{\mathscr{A}}(K) \simeq \bar{A}\left(\mathbb{F}_{K}\right)(c f$. (18) $)$, define

$$
\varphi:\left(\mathscr{Z} / p^{n} \otimes \overline{\mathscr{A}} / p^{n}\right)(k) \rightarrow \bar{A}(\mathbb{F}) / p^{n} ;\{a, x\}_{K / k} \mapsto N_{\mathbb{F}_{K} / \mathbb{F}}(a x)
$$


and

$$
\psi: \bar{A}(\mathbb{F}) / p^{n} \rightarrow\left(\mathscr{Z} / p^{n} \otimes \overline{\mathscr{A}} / p^{n}\right)(k) ; x \mapsto\{1, x\}_{k / k} .
$$

It is easy to see that these maps are well-defined and $\varphi \circ \psi=\mathrm{id}$, where id is the identity map. To show $\psi \circ \varphi=$ id, take an element of the form $\{a, x\}_{K / k} \in\left(\mathscr{Z} / p^{n} \otimes \overline{\mathscr{A}} / p^{n}\right)(k)$. Let $k^{\prime} \subset K$ be the maximal unramified subextension of $K / k$. We have

$$
\begin{aligned}
\psi \circ \varphi\left(\{a, x\}_{K / k}\right) & =\left\{1, N_{\mathbb{F}_{K} / \mathbb{F}}(a x)\right\}_{k / k} \\
& =\left\{a, N_{k^{\prime} / k} x\right\}_{k / k} \quad\left(\text { since } N_{k^{\prime} / k}=N_{\mathbb{F}_{K} / \mathbb{F}}: \overline{\mathscr{A}}(K)=\bar{A}\left(\mathbb{F}_{K}\right) \rightarrow \overline{\mathscr{A}}(k)=\bar{A}(\mathbb{F})\right) \\
& =\left\{\operatorname{Res}_{k^{\prime} / k} a, x\right\}_{k^{\prime} / k} \quad(\text { by the projection formula) } \\
& =\{a, x\}_{k^{\prime} / k} \quad\left(\text { by } \operatorname{Res}_{k^{\prime} / k} a=e\left(k^{\prime} / k\right) a=a\right) \\
& =\left\{N_{K / k^{\prime}} a, x\right\} \quad\left(\text { by } N_{K / k^{\prime}}(a)=f\left(K / k^{\prime}\right) a=a\right) \\
& =\left\{a, \operatorname{Res}_{K / k^{\prime}} x\right\}_{k^{\prime} / k} \quad(\text { by the projection formula) } \\
& =\{a, x\}_{K / k} \quad\left(\operatorname{since}_{\operatorname{Res}_{K / k^{\prime}}}=\mathrm{id}: \overline{\mathscr{A}}\left(k^{\prime}\right)=\bar{A}\left(\mathbb{F}_{K}\right) \rightarrow \overline{\mathscr{A}}(K)=\bar{A}\left(\mathbb{F}_{K}\right)\right) .
\end{aligned}
$$

Claim. $\left(\mathscr{U}_{n} \otimes \overline{\mathscr{A}} / p^{n}\right)(k)=0$.

Proof. Recall that $U_{k}^{1}=1+\mathfrak{m}_{k} \subset U_{k}=O_{k}^{\times}$induces $U_{k} / U_{k}^{1} \simeq \mathbb{F}^{\times}$. The residue field $\mathbb{F}$ is finite, in particular, perfect, so that we have $\mathscr{U}_{n}(k)=\operatorname{Im}\left(U_{k}^{1} \rightarrow k^{\times} / p^{n}\right)$. From the norm arguments, it is enough to show that $\{a, x\}_{k / k}=0$ in $\left(\mathscr{U}_{n} \otimes \mathscr{A} / p^{n}\right)(k)$. For such an element $\{a, x\}_{k / k}$, there exists a finite unramified extension $K / k$ such that $\operatorname{Res}_{K / k}(x)=p^{n} \xi$ for some $\xi \in \overline{\mathscr{A}}(K) \simeq \bar{A}\left(\mathbb{F}_{K}\right)$. Since the norm map $N_{K / k}: U_{K}^{1} \rightarrow U_{k}^{1}$ is surjective ([17], Chap. V, Prop. 3), one can find $\alpha \in \mathscr{U}_{n}(K)$ such that $N_{K / k}(\alpha)=a$. From this, we obtain

$$
\begin{aligned}
\{a, x\}_{k / k} & =\left\{N_{K / k}(\alpha), x\right\}_{k / k} \\
& =\left\{\alpha, \operatorname{Res}_{K / k}(x)\right\}_{K / k} \quad \text { (by the projection formula) } \\
& =\left\{\alpha, p^{n} \xi\right\}_{K / k}=0 .
\end{aligned}
$$

The short exact sequence (20) and the above claims yield the assertion.

\section{Mackey product and the Somekawa $K$-group}

We define

$$
N:=\max \left\{n \in \mathbb{Z}_{\geq 0} \mid A\left[p^{n}\right] \subset A(k)\right\} .
$$

In the following, we assume the abelian variety $A$ satisfies the condition (Rat) in the last section. From this assumption (Rat), $N \geq 1$. We fix isomorphisms $A\left[p^{n}\right] \simeq\left(\mu_{p^{n}}\right)^{\oplus 2 g}$ for all $n \leq N$ as 
follows: First, take an isomorphism $A\left[p^{N}\right] \simeq\left(\mu_{p^{N}}\right)^{\oplus 2 g}$ which makes the diagram

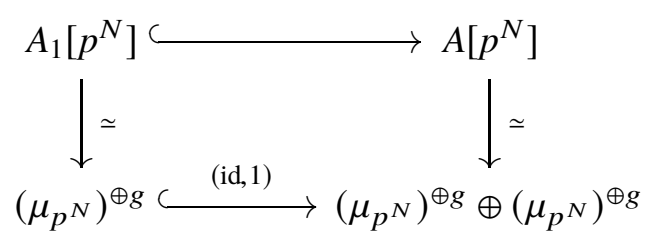

commutes as in (12). For each $1 \leq n<N$, we choose $A\left[p^{n}\right] \simeq\left(\mu_{p^{n}}\right)^{\oplus 2 g}$ as the following diagram is commutative:

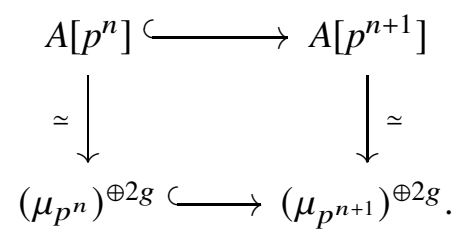

To simplify the notation, put

$$
\mathscr{M}_{n}:=\left(\mathbb{G}_{m} \otimes A\right)(k) / p^{n} \simeq\left(\mathbb{G}_{m} / p^{n} \otimes A / p^{n}\right)(k), \quad \text { and } \quad \mathscr{H}_{n}:=H^{2}\left(k, \mu_{p^{n}} \otimes A\left[p^{n}\right]\right) .
$$

Theorem 4.2. For any $n \geq 1$, the Galois symbol map $s_{p^{n}}^{M}: \mathscr{M}_{n} \rightarrow \mathscr{H}_{n}($ Def. 2.3) is bijective.

Proof. The map $s_{p^{n}}^{M}$ is surjective ([4], Thm. A.1). We show that $s_{p^{n}}^{M}$ is injective by induction on $n$.

Claim. $s_{p}^{M}$ is bijective.

Proof. By the fixed isomorphism $A[p] \simeq\left(\mu_{p}\right)^{\oplus 2 g}$ as in (22), we have the isomorphism

$$
A / p \simeq \mathscr{U}^{\oplus g} \oplus \mathscr{V}^{\oplus g}
$$

of Mackey functors (Cor. 3.4). The Mackey product $\mathscr{M}_{1}=\mathbb{G}_{m} / p \otimes A / p$ is decomposed as

$$
\mathscr{M}_{1}=\mathbb{G}_{m} / p \otimes\left(\mathscr{U}^{\oplus g} \oplus \mathscr{V}^{\oplus g}\right) \simeq\left(\mathbb{G}_{m} / p \otimes \mathscr{U}\right)^{\oplus g} \oplus\left(\mathbb{G}_{m} / p \otimes \mathscr{V}\right)^{\oplus g} .
$$

Since the composition

$$
\left(\mathbb{G}_{m} / p \otimes \mathscr{V}\right)(k) \rightarrow\left(\mathbb{G}_{m} / p \otimes \mathscr{U}\right)(k) \rightarrow\left(\mathbb{G}_{m} / p \otimes \mathbb{G}_{m} / p\right)(k) \simeq K_{2}^{M}(k) / p \simeq H^{2}\left(k, \mu_{p}^{\otimes 2}\right)
$$

is bijective ([7], Thm. 3.6), the Galois symbol map $s_{p}^{M}$ is also bijective from the commutative diagram below:

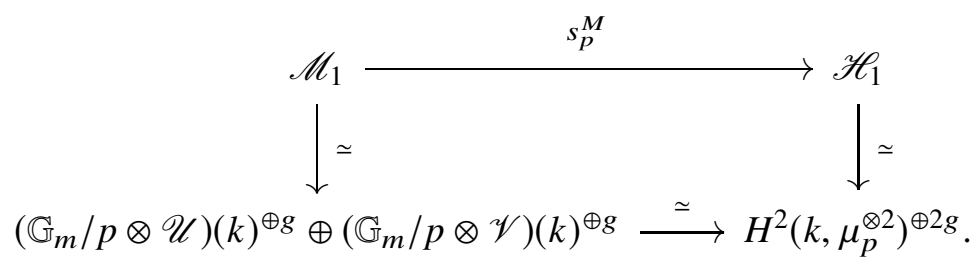


We consider the following commutative diagram with exact rows except possibly at $\mathscr{M}_{n}$ (cf. [14], Proof of Lem. 4.2.2):

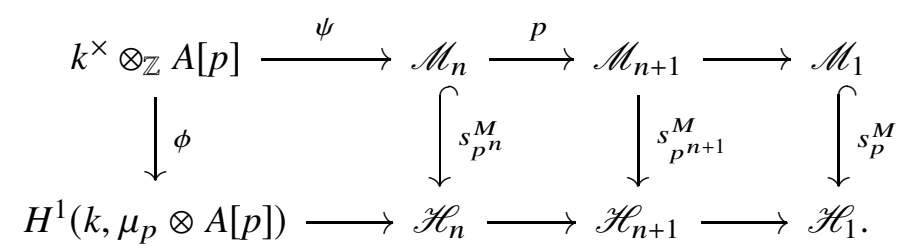

Here, the far left vertical map $\phi$ is given by

$$
k^{\times} \otimes_{\mathbb{Z}} A[p] \stackrel{\delta_{\mathfrak{G}_{m}} \otimes \mathrm{id}}{\longrightarrow} H^{1}\left(k, \mu_{p}\right) \otimes_{\mathbb{Z}} H^{0}(k, A[p]) \stackrel{\cup}{\rightarrow} H^{1}\left(k, \mu_{p} \otimes A[p]\right)
$$

and $\psi$ is induced from $A[p] \hookrightarrow A(k) \rightarrow A(k) / p^{n}$. By the fixed isomorphism $A[p] \simeq\left(\mu_{p}\right)^{\oplus 2 g}$ of trivial Galois modules, the map $\phi$ becomes

$$
k^{\times} \otimes_{\mathbb{Z}} A[p] \rightarrow\left(k^{\times} / p \otimes_{\mathbb{Z}} \mu_{p}\right)^{\oplus 2 g} \simeq H^{1}\left(k, \mu_{p}^{\otimes 2}\right)^{\oplus 2 g} \simeq H^{1}\left(k, \mu_{p} \otimes A[p]\right) .
$$

In particular, $\phi$ is surjective. From the diagram chase and the induction hypothesis, $s_{p^{n+1}}^{M}$ is injective.

Corollary 4.3. (i) For any $n \geq 1$, we have $\mathscr{M}_{n}=\left(\mathbb{G}_{m} / p^{n} \otimes A / p^{n}\right)(k) \simeq K\left(k ; \mathbb{G}_{m}, A\right) / p^{n}$.

(ii) For any $n \geq 1$, the Galois symbol map $s_{p^{n}}$ is bijective.

(iii) For any $n \leq N$, we have $\mathscr{M}_{n} \simeq\left(\mathbb{Z} / p^{n}\right)^{\oplus 2 g}$.

Proof. As $s_{p^{n}}^{M}$ factors through $s_{p^{n}}$, the assertions (i) and (ii) follow from Theorem 4.2, If $n \leq N$, we have

$$
K\left(k ; \mathbb{G}_{m}, A\right) / p^{n} \stackrel{\simeq}{\rightarrow} \mathscr{H}_{n} \simeq H^{2}\left(k, \mu_{p^{n}}^{\otimes 2}\right)^{\oplus 2 g} \simeq\left(\mathbb{Z} / p^{n}\right)^{\oplus 2 g} .
$$

The assertion (iii) follows from (i).

Note that we have $\mathscr{M}_{n} \simeq K\left(k ; \mathbb{G}_{m}, A\right) / p^{n}$ the middle vertical map in the diagram (19) is bijective (Cor.4.3) and hence we have $\overline{\mathscr{M}}_{n} \simeq \overline{\mathscr{K}}_{n}$.

Corollary 4.4. $\overline{\mathscr{K}}_{n} \simeq \overline{\mathscr{M}}_{n} \simeq \bar{A}(\mathbb{F}) / p^{n}$.

Proof. The latter isomorphism $\overline{\mathscr{M}}_{n} \simeq \bar{A}(\mathbb{F}) / p^{n}$ follows from Lemma 4.1.

\section{Formal groups}

Since the Mackey functor $\widehat{A}$ defined by the formal group of $A$ satisfies the Galois descent, we have the Galois symbol map (Def. 2.3) of the form

$$
\widehat{s}_{p^{n}}^{M}: \widehat{\mathscr{M}}_{n} \simeq\left(\mathbb{G}_{m} \otimes \widehat{A}\right)(k) / p^{n} \rightarrow H^{2}\left(k, \mu_{p^{n}} \otimes \widehat{A}\left[p^{n}\right]\right)=: \widehat{\mathscr{H}}_{n} .
$$


Lemma 4.5. For $n \leq N$, we have the following:

(i) The Galois symbol map $\widehat{s}_{p^{n}}^{M}: \widehat{\mathscr{M}}_{n} \rightarrow \widehat{\mathscr{H}}_{n}$ is bijective.

(ii) $\widehat{\mathscr{M}_{n}} \simeq \widehat{\mathscr{K}_{n}} \simeq\left(\mathbb{Z} / p^{n}\right)^{\oplus g}$.

Proof. (i) Consider the following commutative diagram with exact rows:

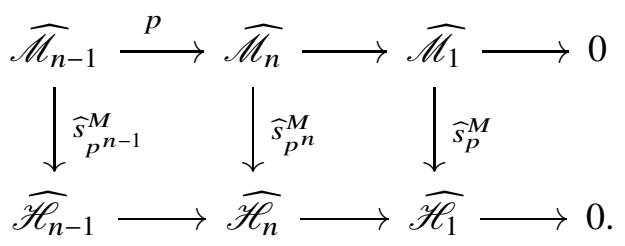

From the assumption $n \leq N$, we have $\widehat{\mathscr{H}}_{n} \simeq H^{2}\left(k, \mu_{p^{n}}^{\otimes 2}\right)^{\oplus g} \simeq\left(\mathbb{Z} / p^{n}\right)^{\oplus g}$. By counting the orders, the left lower map in (23) is injective. By induction on $n$, it is enough to show that $\widehat{s}_{p}^{M}: \widehat{\mathscr{M}}_{1} \rightarrow \widehat{\mathscr{H}}_{1}$ is bijective. In this case, we have an isomorphism

$$
\widehat{A} / p \stackrel{\simeq}{\rightarrow} \mathscr{U}^{\oplus g}
$$

of Mackey functors (Cor. 3.4). On the other hand, we have $\widehat{\mathscr{H}_{1}} \simeq H^{2}\left(k, \mu_{p}^{\otimes 2}\right)^{\oplus g}$. The assertion now reduced to showing that the composition

$$
\left(\mathbb{G}_{m} / p \otimes \mathscr{U}\right)(k) \rightarrow\left(\mathbb{G}_{m} / p \otimes \mathbb{G}_{m} / p\right)(k) \stackrel{\simeq}{\rightarrow} H^{2}\left(k, \mu_{p}^{\otimes 2}\right)
$$

is bijective. This assertion follows from [7], Theorem 3.6 (i).

(ii) The Galois symbol map $s_{p^{n}}: K\left(k ; \mathbb{G}_{m}, A\right) / p^{n} \rightarrow \mathscr{H}_{n}=H^{2}\left(k, \mu_{p^{n}} \otimes A\left[p^{n}\right]\right)$ is bijective from Theorem 4.2 and $\mathscr{H}_{n} \simeq H^{2}\left(k, \mu_{p^{n}}^{\otimes 2}\right)^{\oplus 2 g} \simeq\left(\mathbb{Z} / p^{n}\right)^{\oplus 2 g}$. From the fixed commutative diagrams in (22), we have the following commutative diagram:

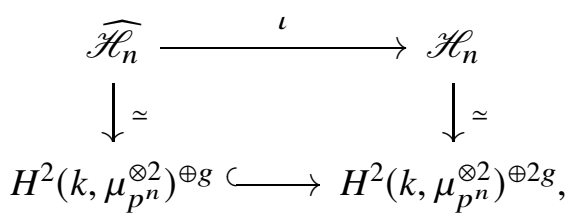

where $\widehat{\mathscr{H}_{n}}=H^{2}\left(k, \mu_{p^{n}} \otimes \widehat{A}\left[p^{n}\right]\right)$. As the bottom horizontal map is the inclusion map, the map $\iota$ above is injective. Next, we consider the commutative diagram extended from (19):

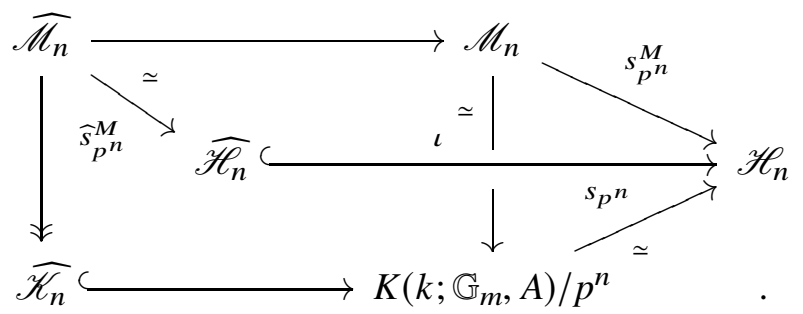


Since $\widehat{s}_{p^{n}}^{M}: \widehat{\mathscr{M}}_{n} \rightarrow \widehat{\mathscr{H}}_{n}$ is bijective, the left vertical map in the above diagram is bijective. Therefore, the induced map $\widehat{\mathscr{K}}_{n} \rightarrow \widehat{\mathscr{H}}_{n}$ is also bijective.

Proposition 4.6. We further assume the following condition:

(Ram) $k\left(\mu_{p^{N+1}}\right) / k$ is a non-trivial and totally ramified extension.

Then, we have $\widehat{\mathscr{M}_{n}} \simeq \widehat{\mathscr{K}_{n}} \simeq\left(\mathbb{Z} / p^{\min \{N, n\}}\right)^{\oplus g}$ for any $n \geq 1$.

Proof. From Lemma4.5, we may assume $n \geq N$. First, we show the following claim:

Claim (cf. [5], Proof of Thm. 3.14). Fix $\zeta \in \mu_{p^{N}}$ a primitive $p^{N}$-th root of unity. Then, $\widehat{\mathscr{M}_{1}}=\left(\mathbb{G}_{m} / p \otimes \widehat{A} / p\right)(k)$ is generated by elements of the form $\{\zeta, w\}_{k / k}$, where $w \in \widehat{A}(k) / p$.

Proof. Recall that the Hilbert symbol (-,-) : $k^{\times} \otimes k^{\times} \rightarrow \mu_{p} \simeq \mathbb{Z} / p$ satisfies

$$
(x, y)=0 \Leftrightarrow y \in N_{k(\sqrt[R]{x}) / k}\left(k(\sqrt[p]{x})^{\times}\right), \quad \text { for } x, y \in k^{\times}
$$

(cf. [22], Prop. 4.3). From the assumption (Ram), there exists $y \in \bar{U}_{k}=\operatorname{Im}\left(U_{k} \rightarrow k^{\times} / p\right)$ such that $(\zeta, y) \neq 0$. In fact, putting $K=k(\sqrt[p]{\zeta})=k\left(\mu_{p^{N+1}}\right)$, we have $U_{k} / N_{K / k} U_{K} \simeq k^{\times} / N_{K / k} K^{\times}$ (cf. the proof of [17], Chap. V, Sect. 3, Cor. 7) and local class field theory says $k^{\times} / N_{K / k} K^{\times} \simeq$ $\operatorname{Gal}(K / k) \neq 0$ (cf. [17], Chap. XIII, Sect. 3). Thus, there exists $y \in U_{k} \backslash N_{K / k} U_{K}$ such that $(\zeta, y) \neq 0$ from (25). As $(\zeta, y) \neq 0$, the chosen element $y$ is non-trivial in $\bar{U}_{k}$. We use the same notation $y$ as an element in $\bar{U}_{k}$. For each $1 \leq i \leq g$, put

$$
y^{(i)}:=(1, \ldots, 1, \stackrel{i}{y}, 1, \ldots, 1) \in\left(\bar{U}_{k}\right)^{\oplus g}
$$

and we denote by $w^{(i)} \in \widehat{A}(k) / p$ the element corresponding to $y^{(i)}$ through the isomorphism $\widehat{A}(k) / p \simeq\left(\bar{U}_{k}\right)^{\oplus g}$ as in (24). The Galois symbol map is compatible with the Hilbert symbol map ([17], Chap. XIV, Sect. 2, Prop. 5) as the following commutative diagram indicates:

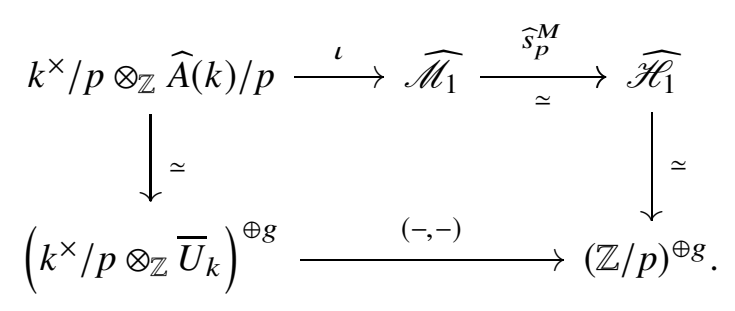

Here, we identify the isomorphism $H^{2}\left(k, \mu_{p}^{\otimes 2}\right) \simeq \mathbb{Z} / p$, the map $\widehat{s}_{p}^{M}$ is bijective (Thm. 4.2), and the map $\iota$ is given by $\iota(x \otimes w)=\{x, w\}_{k / k}$. The image of $\zeta \otimes w^{(i)} \in k^{\times} / p \otimes_{\mathbb{Z}} \widehat{A}(k) / p$ in $(\mathbb{Z} / p)^{\oplus g}$ via the lower left corner in (26) is

$$
\xi^{(i)}:=\left(0, \ldots, 0,\left(\zeta_{\zeta}^{\vee}, y\right), 0, \ldots, 0\right) \in(\mathbb{Z} / p)^{\oplus g} .
$$


These elements $\xi^{(i)}(1 \leq i \leq g)$ generate $(\mathbb{Z} / p)^{\oplus g}$ and hence the symbols $\left\{\zeta, w^{(i)}\right\}_{k / k}=\iota\left(\zeta \otimes w^{(i)}\right)$ $(1 \leq i \leq g)$ generate $\widehat{\mathscr{M}}_{1}$.

There is a short exact sequence

$$
\widehat{\mathscr{M}}_{1} p^{n} \rightarrow \widehat{\mathscr{M}}_{n+1} \rightarrow \widehat{\mathscr{M}}_{n} \rightarrow 0
$$

where the first map is given by the multiplication by $p^{n}$. By Lemma 4.5, we have $\widehat{\mathscr{K}}_{N} \simeq$ $\left(\mathbb{Z} / p^{N}\right)^{\oplus g}$. From the above claim and the induction on $n \geq N$, we have $\widehat{\mathscr{M}}_{n+1} \simeq \widehat{\mathscr{M}}_{n} \simeq\left(\mathbb{Z} / p^{N}\right)^{\oplus g}$. The surjective homomorphisms

$$
\widehat{\mathscr{M}}_{n} \rightarrow \widehat{\mathscr{K}}_{n} \rightarrow \widehat{\mathscr{K}}_{N} \simeq\left(\mathbb{Z} / p^{N}\right)^{\oplus g}
$$

induce $\widehat{\mathscr{K}_{n}} \simeq\left(\mathbb{Z} / p^{N}\right)^{\oplus g}$.

Remark 4.7. In the case where $A=E$ is an elliptic curve with (Ord), we can show Proposition 3.6 by the essentially same proof, under the condition

(Ram') $k\left(\widehat{E}\left[p^{N+1}\right]\right) / k$ is a non-trivial extension

instead of (Ram).

\section{Proof of the main theorem}

Theorem 4.8. Let $A$ be an abelian variety over $k$ of dimension $g$. We assume the condition (Ord), (Rat) and (Ram) (in Prop. 4.6). Then, we have

$$
K\left(k ; \mathbb{G}_{m}, A\right)_{\mathrm{fin}} \simeq\left(\mathbb{Z} / p^{N}\right)^{\oplus g} \oplus \bar{A}(\mathbb{F}) .
$$

Proof. For each $m$ prime to $p$, we have $K\left(k ; \mathbb{G}_{m}, A\right) / m \simeq \bar{A}(\mathbb{F}) / m$ (Prop.2.6).

Claim. $K\left(k ; \mathbb{G}_{m}, A\right) / p^{n} \simeq \widehat{\mathscr{K}}_{n} \oplus \overline{\mathscr{K}}_{n}$ for each $n \geq 1$.

Proof. The assertion is true for $n \leq N$ by Theorem 4.2 and Lemma4.5, For $n>N$, consider the following diagram:

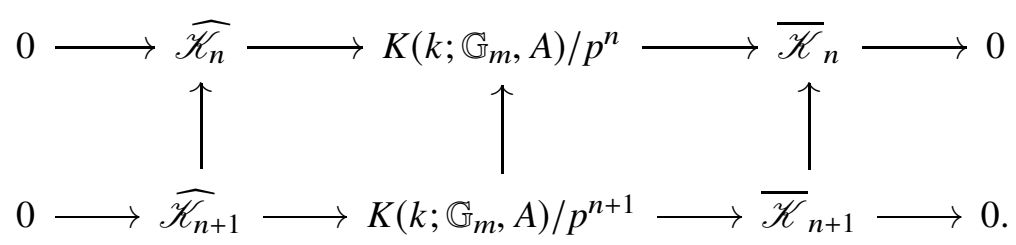

By the induction hypothesis, the top sequence splits. From Proposition 4.6, the left vertical map is bijective so that the lower sequence also splits. 
From Cororally 4.4 and Proposition 4.6, we obtain

$$
K\left(k ; \mathbb{G}_{m}, A\right) / p^{n} \simeq\left(\mathbb{Z} / p^{\min \{n, N\}}\right)^{\oplus g} \oplus \bar{A}(\mathbb{F}) / p^{n}
$$

for any $n$. By taking the limit $\lim _{\longleftarrow}$, we have $K\left(k ; \mathbb{G}_{m}, A\right)_{\text {fin }} \simeq\left(\mathbb{Z} / p^{N}\right)^{\oplus g} \oplus \bar{A}(\mathbb{F})$.

Applying the above theorem to the Jacobian variety $\operatorname{Jac}(X)$, we obtain the following corollary as noted in Introduction.

Corollary 4.9. Let $X$ be a projective smooth curve over $k$ with $X(k) \neq \emptyset$. We assume the conditions (Rat), (Ord), and (Ram) for the Jacobian variety $J=\operatorname{Jac}(X)$ associated to $X$. Then, we have

$$
V(X)_{\text {fin }} \simeq\left(\mathbb{Z} / p^{N}\right)^{\oplus g} \oplus \bar{J}(\mathbb{F}),
$$

where $g=\operatorname{dim} J$ and $N=\max \left\{n \mid J\left[p^{n}\right] \subset J(k)\right\}$.

\section{A. Albanese kernel}

In this appendix, we compute the Albanese kernel for the product of curves over $k$ as a generalization of [21]. Let $A$ be an abelian variety over $k$ assuming (Ord) and (Rat). For $n \geq 1$ with $A\left[p^{n}\right] \subset A(k)$, we choose an isomorphism

$$
A\left[p^{n}\right] \stackrel{\simeq}{\rightarrow}\left(\mu_{p^{n}}\right)^{\oplus 2 g}
$$

of (trivial) Galois modules as in (12). By the same proof as in Prop 3.1, the image of the Kummer map $\delta_{A}: A(k) / p^{n} \rightarrow H^{1}\left(k, A\left[p^{n}\right]\right) \simeq H^{1}\left(k, \mu_{p^{n}}^{\oplus 2 g}\right) \simeq\left(k^{\times} / p^{n}\right)^{\oplus 2 g}$ is determined as follows:

Proposition A.1. For any $n \geq 1$ with $A\left[p^{n}\right] \subset A(K)$ and a finite extension $K / k$, we have

$$
A(K) / p^{n} \stackrel{\simeq}{\rightarrow}\left(\bar{U}_{K}\right)^{\oplus g} \oplus \operatorname{Ker}\left(j: K^{\times} / p^{n} \rightarrow\left(K^{\mathrm{ur}}\right)^{\times} / p^{n}\right)^{\oplus g},
$$

where $j$ is the map induced from the inclusion $K^{\times} \hookrightarrow\left(K^{\mathrm{ur}}\right)^{\times}$.

The above isomorphism is extend to the isomorphism

$$
A / p^{n} \simeq \mathscr{U}^{\oplus g} \oplus \mathscr{V}^{\oplus g}
$$

of Mackey functors, where $\mathscr{U}, \mathscr{V}$ is the sub Mackey functors of $\mathbb{G}_{m} / p^{n}$ defined by

$$
\mathscr{U}(K):=\operatorname{Im}\left(U_{K} \rightarrow K^{\times} / p^{n}\right), \quad \mathscr{V}(K):=\operatorname{Ker}\left(j: K^{\times} / p^{n} \rightarrow\left(K^{\mathrm{ur}}\right)^{\times} / p^{n}\right),
$$

for any finite extension $K / k$ ( $c f$. Cor. 3.4). Now we use the following notation:

- $X_{i}$ : smooth projective curves over $k$ with $k$-rational point $X_{i}(k) \neq \emptyset$ for $i=1,2$, 
- $J_{i}:=\operatorname{Jac}\left(X_{i}\right)$ : the Jacobian variety associated to $X_{i}$ of dimension $g_{i}$.

The kernel of the degree map deg: $\mathrm{CH}_{0}\left(X_{1} \times X_{2}\right) \rightarrow \mathbb{Z}$ is denoted by $A_{0}\left(X_{1} \times X_{2}\right)$. The kernel $T\left(X_{1} \times X_{2}\right)$ of the Albanese map

$$
\text { alb }: A_{0}\left(X_{1} \times X_{2}\right) \rightarrow \operatorname{Alb}_{X_{1} \times X_{2}}(k)=J_{1}(k) \oplus J_{2}(k)
$$

which is called the Albanese kernel is also written by the Somekawa $K$-group as

$$
T\left(X_{1} \times X_{2}\right) \simeq K\left(k ; J_{1}, J_{2}\right)
$$

([14]). From the same computation as in [21], Theorem 4.1, we recover [3], Corollary 8.9.

Corollary A.2. Assume that $J_{1}, J_{2}$ satisfy (Ord) and all $p^{n}$-torsion points of $J_{1}$ and $J_{2}$ are k-rational. Then, we have

$$
T\left(X_{1} \times X_{2}\right) / p^{n} \simeq\left(\mathbb{Z} / p^{n}\right)^{\oplus g_{1} g_{2}},
$$

where $g_{i}=\operatorname{dim} J_{i}$.

Proof. From $K\left(k ; J_{1}, J_{2}\right) \simeq T\left(X_{1} \times X_{2}\right)$, it is enough to show $K\left(k ; J_{1}, J_{2}\right) / p^{n} \simeq\left(\mathbb{Z} / p^{n}\right)^{\oplus g_{1} g_{2}}$. We fix isomorphisms $J_{i}\left[p^{n}\right] \simeq\left(\mu_{p^{n}}\right)^{\oplus 2 g_{i}}$ of (trivial) Galois modules as above. We have

$$
J_{1} / p^{n} \otimes J_{2} / p^{n} \simeq((\mathscr{U} \otimes \mathscr{U}) \oplus(\mathscr{U} \otimes \mathscr{V}) \oplus(\mathscr{V} \otimes \mathscr{U}) \oplus(\mathscr{V} \otimes \mathscr{V}))^{\oplus g_{1} g_{2}} .
$$

The Galois symbol maps give the following commutative diagram:

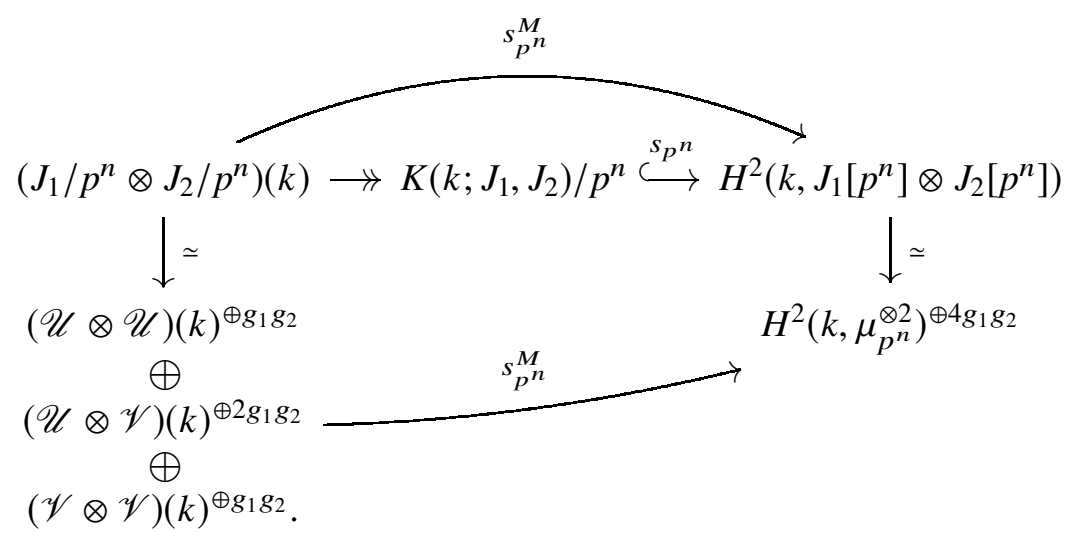

Here, $s_{p^{n}}: K\left(k ; J_{1}, J_{2}\right) / p^{n} \rightarrow H^{2}\left(k, J_{1}\left[p^{n}\right] \otimes J_{2}\left[p^{n}\right]\right)$ is injective ([14], Rem. 4.5 .8 (b)), and the bottom map is given by composing $s_{p^{n}}^{M}:\left(\mathbb{G}_{m} / p^{n} \otimes \mathbb{G}_{m} / p^{n}\right)(k) \rightarrow H^{2}\left(k, \mu_{p^{n}}^{\otimes 2}\right)$ (Def. 2.3) after the natural map $(\mathscr{U} \otimes \mathscr{U})(k) \rightarrow\left(\mathbb{G}_{m} / p^{n} \otimes \mathbb{G}_{m} / p^{n}\right)(k),(\mathscr{U} \otimes \mathscr{V})(k) \rightarrow\left(\mathbb{G}_{m} / p^{n} \otimes \mathbb{G}_{m} / p^{n}\right)(k)$, or $(\mathscr{V} \otimes \mathscr{V})(k) \rightarrow\left(\mathbb{G}_{m} / p^{n} \otimes \mathbb{G}_{m} / p^{n}\right)(k)$. The Galois symbol map is written by the Hilbert symbol ([17], Chap. XIV, Sect. 2, Prop. 5) as follows: 


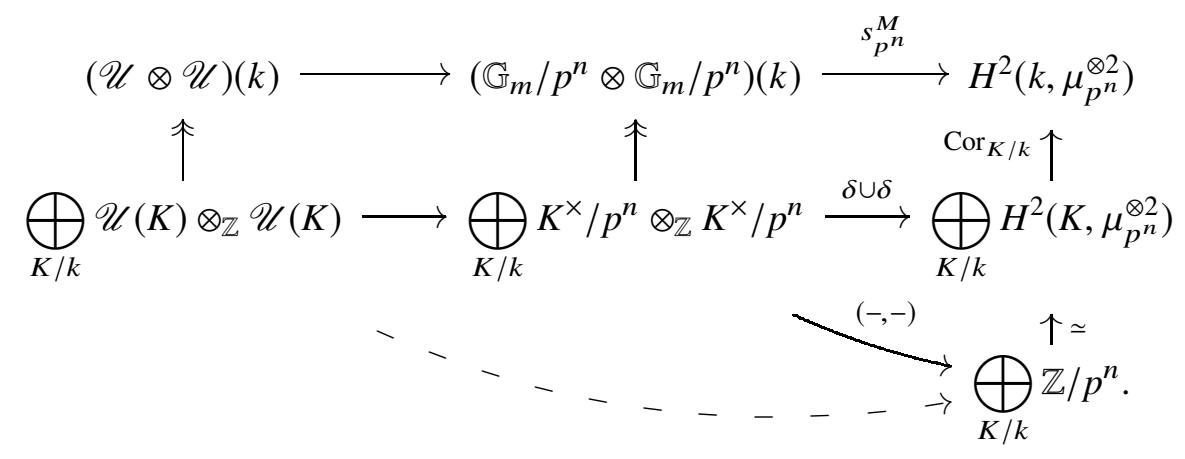

For each finite extension $K / k$, the image of $\mathscr{U}(K) \otimes_{\mathbb{Z}} \mathscr{U}(K)$ by the Hilbert symbol (the dotted arrow in the above diagram) is isomorphic to $\mathbb{Z} / p^{n}$ ([21], Prop. 2.5) so that

$$
s_{p^{n}}^{M}((\mathscr{U} \otimes \mathscr{U})(k)) \simeq \mathbb{Z} / p^{n} .
$$

On the other hand, the image of $\mathscr{U}(K) \otimes_{\mathbb{Z}} \mathscr{V}(K)$ and $\mathscr{V}(K) \otimes_{\mathbb{Z}} \mathscr{V}(K)$ by the Hilbert symbol is trivial and hence

$$
s_{p^{n}}^{M}((\mathscr{U} \otimes \mathscr{V})(k))=s_{p^{n}}^{M}((\mathscr{V} \otimes \mathscr{V})(k))=0 .
$$

From the above diagram (27), these computations imply

$$
s_{p^{n}}^{M}\left(\left(J_{1} / p^{n} \otimes J_{2} / p^{n}\right)(k)\right) \simeq K\left(k ; J_{1}, J_{2}\right) / p^{n} \simeq\left(\mathbb{Z} / p^{n}\right)^{\oplus g_{1} g_{2}} .
$$

\section{References}

[1] S. Bloch, Algebraic K-theory and classfield theory for arithmetic surfaces, Ann. of Math. (2) 114 (1981), no. 2, 229-265.

[2] The Sage Developers, Sagemath, the Sage Mathematics Software System (Version 7.4), 2016, http://www. sagemath.org.

[3] E. Gazaki, A finer Tate duality theorem for local Galois symbols, J. Algebra 509 (2018), $337-385$.

[4] _ A Tate duality theorem for local Galois symbols II; The semi-abelian case, J. Number Theory 204 (2019), 532-560.

[5] E. Gazaki and I. Leal, Zero cycles on a product of elliptic curves over a p-adic field, arXiv:1805.05496.

[6] M. Hindry and J. H. Silverman, Diophantine geometry: An introduction, Graduate Texts in Mathematics, vol. 201, Springer-Verlag, New York, 2000. 
[7] T. Hiranouchi, Milnor K-groups attached to elliptic curves over a p-adic field, Funct. Approx. Comment. Math. 54 (2016), no. 1, 39-55.

[8] B. Kahn, The decomposable part of motivic cohomology and bijectivity of the norm residue homomorphism, Algebraic $K$-theory, commutative algebra, and algebraic geometry (Santa Margherita Ligure, 1989), Contemp. Math., vol. 126, Amer. Math. Soc., Providence, RI, 1992, pp. 79-87.

[9] N. M. Katz and S. Lang, Finiteness theorems in geometric classfield theory, Enseign. Math. (2) 27 (1981), no. 3-4, 285-319 (1982), With an appendix by Kenneth A. Ribet.

[10] A. Mattuck, Abelian varieties over p-adic ground fields, Ann. of Math. (2) 62 (1955), 92-119.

[11] B. Mazur, Rational points of abelian varieties with values in towers of number fields, Invent. Math. 18 (1972), 183-266.

[12] A. S. Merkurjev and A. A. Suslin, $K$-cohomology of Severi-Brauer varieties and the norm residue homomorphism, Izv. Akad. Nauk SSSR Ser. Mat. 46 (1982), no. 5, 1011-1046, 1135-1136.

[13] J. Neukirch, A. Schmidt, and K. Wingberg, Cohomology of number fields, second ed., Grundlehren der Mathematischen Wissenschaften [Fundamental Principles of Mathematical Sciences], vol. 323, Springer-Verlag, Berlin, 2008.

[14] W. Raskind and M. Spiess, Milnor K-groups and zero-cycles on products of curves over p-adic fields, Compositio Math. 121 (2000), 1-33.

[15] S. Saito, Class field theory for curves over local fields, J. Number Theory 21 (1985), no. 1, 44-80.

[16] _ Unramified class field theory of arithmetical schemes, Ann. of Math. (2) 121 (1985), no. 2, 251-281.

[17] J.-P. Serre, Corps locaux, Hermann, Paris, 1968, Deuxième édition, Publications de l'Université de Nancago, No. VIII.

[18] J. H. Silverman, The arithmetic of elliptic curves, second ed., Graduate Texts in Mathematics, vol. 106, Springer, Dordrecht, 2009.

[19] M. Somekawa, On Milnor K-groups attached to semi-abelian varieties, $K$-Theory 4 (1990), no. $2,105-119$.

[20] M. Spiess and T. Yamazaki, A counterexample to generalizations of the Milnor-Bloch-Kato conjecture, J. K-Theory 4 (2009), no. 1, 77-90. MR 2538717 (2010i:19008)

[21] T. Takemoto, Zero-cycles on products of elliptic curves over p-adic fields, Acta Arith. 149 (2011), no. 3, 201-214. MR 2812424 
[22] J. Tate, Relations between $K_{2}$ and Galois cohomology, Invent. Math. 36 (1976), 257-274.

[23] T. Yamazaki, On Chow and Brauer groups of a product of Mumford curves, Math. Ann. 333 (2005), 549-567.

[24] _ Class field theory for a product of curves over a local field, Math. Z. 261 (2009), no. 1, 109-121. 